\title{
Effect of Hydro and Hormonal Priming on Growth and Development of Rice under Timely and Late Sown Conditions
}

\author{
Mahesh Kumar*, Rajesh Kumar Singhal, Bandana Bose
}

Department of Plant Physiology, Institute of Agricultural Sciences, Banaras Hindu University, Varanasi- 221005, India

*Corresponding author

Keywords

Priming, Growth and Development of

Rice, Late Sown

Conditions

Article Info

Accepted:

20 April 2018

Available Online:

10 May 2018
In the present investigation HUR 105 genotype was selected to see the effect of hydro and hormonal seed priming on growth and development of rice crop. Priming of seeds has been done with distilled water (hydro) and two hormones (Kinetin and salicylic acid) and different growth parameters, such as plant height, number of tillers hill ${ }^{-1}$, number of green leaves hill ${ }^{-1}$, leaf area hill ${ }^{-1}$ and leaf area index were studied at different study periods under timely and late sown conditions. Primed seeds increased all the growth parameters in respect to non-primed (control) sets.

\section{Introduction}

Rice is a most important cereal crop that provides food to most of the Asian population. World population is increasing day by day followed by increasing food demand. Proper sowing time and method of sowing are the most important growth and yield deciding factors that allow the crop to complete its life phase timely and successfully under a specific agro-ecology (Baloch et al., 2006). Various seed priming techniques including hydro, halo, hormonal, nutrient and chemical priming are employed in rice. In rice crop, seed priming provides a vigorous start that typically exhibit faster and uniform emergence, accomplish better seedling establishment, and gives high yields (Lichtfouse 2009).Seed priming enhanced germination performance and increased seedling vigor under both optimal and stress environments in diverse species, such as soybean(Glycine max), maize (Zea mays), pepper (Capsicum annuum), spinach (Spinacea oleracea), wheat (Triticum aestivum), rice (Oryza sativa) and mustard (Brassica juncea). (Iqbal and Ashraf 2007, Bose et al., 2007, Farooq et al., 2008, Krishnottar et al., 2009, Korkmaz and Korkmaz, 2009, Zhuo et al., 2009, Chen et al., 2011, Srivastava and Bose 2012, Pant and Bose 2016, Kumar et al., 2016). 


\section{Materials and Methods}

In the present piece of work rice variety HUR 105 was selected for experimental purpose. Healthy and bold seeds were surface sterilized by keeping them in $0.01 \% \mathrm{HgCl}_{2}$ solution for 5 minutes and then thoroughly washed with distilled water for 5-6 times and two priming treatments were used for this experiment included hydropriming $\left(\mathrm{T}_{1}\right)$ (using distilled water) and hormonal priming using $5 \mathrm{ppm}$ concentration of Kinetin $\left(\mathrm{T}_{2}\right)$ and $0.75 \mathrm{mM}$ concentration of salicylic acid $\left(\mathrm{T}_{3}\right)$ for $18 \mathrm{~h}$ with a set of non primed $\left(\mathrm{T}_{0}\right.$; control) seeds. After $18 \mathrm{~h}$ these seeds were air dried by fan to bring back to its original moisture content; further the seeds were kept in paper packets and used within one month. Sowing of seeds was done at two different conditions i.e. timely (recommended) and late (20 Days delay after recommended sowing). Experiments were conducted in Factorial Randomized Block Design (RBD) during 2015 and 2016 at Agricultural Research Farm, Banaras Hindu University, Varanasi.

\section{Plant height (cm)}

Height of the plant was measured from ground to tip of the largest leaf with the help of scale. The average plant height was calculated by taking the mean of three observations and expressed in centimeter.

\section{Number of tillers hill ${ }^{-1}$}

Number of tillers hill ${ }^{-1}$ was counted at 20, 40, 60 and 80 days after transplanting (DAT). For this purpose, $3^{\text {rd }}$ row from each plot were selected and average values were computed from the recorded observation.

\section{Number of green leaves hill ${ }^{-1}$}

The number of green leaves hill ${ }^{-1}$ was counted at 20, 40, 60 and 80 DAT manually.

\section{Leaf area hill ${ }^{-1}$}

Leaf area hill ${ }^{-1}$ was measured with the help of CL-202 portable laser leaf area meter (CID Bio science).

\section{Leaf area index}

Leaf area index of rice crop was calculated by the method of Watson (1947) as follows.

Leaf Area index $(\mathrm{LAI})=\begin{gathered}\text { Leaf area } \\ ----------- \\ \text { Ground area }\end{gathered}$

\section{Statistical analysis}

Mean values were taken from each treatment of three independent replications. Analysis of variance was performed by using SPSS version 16.0 software.

Duncan's test was used to determine significant difference among treatments.

\section{Results and Discussion}

\section{Plant height (cm)}

Table1 represents plant height at 20,40,60, 80 and100 DAT which was obtained from primed and non primed seeds of rice. Plant height was found to be significantly affected by the sowing time and reduction in plant height was noted at late sown condition.

The seed primed with hydro $\left(T_{1}\right)$, Kinetin $\left(T_{2}\right)$ and Salicylic acid $\left(\mathrm{T}_{3}\right)$ were found to maintain significant higher plant height Seed primed with kinetin $\left(\mathrm{T}_{2}\right)$ showed maximum plant height followed by $\mathrm{T}_{3}$ and $\mathrm{T}_{1}$ in comparison to nonprimed control set $\left(\mathrm{T}_{0}\right)$.

The similar trend was recorded in subsequent period and in both the years, timely as well as late sown condition. 


\section{Number of tillers hill ${ }^{-1}$}

Table 2 represents the number of tillers hill ${ }^{-1}$ at 20, 40, 60, 80 and 100 DAT. Results depicted that number of tillers were found to increase with increasing age till 60 DAT, after that it was started decline. The maximum number of tillers hill ${ }^{-1}$ were recorded in kinetin primed set $\left(T_{2}\right)$ followed by $T_{1}$ and $T_{3}$ over non primed set $\mathrm{T}_{0}$. However in late sown condition reduction in tillers numbers were noted. The trend was found similar for both the studied years and all studied periods. The data regarding number of tillers hill $^{-1}$ was found statistically significant.

\section{Number of green leaves hill ${ }^{-1}$}

Table 3 represents number of green leaves hill-
1, was recorded at 20, 40, 60 and 80 DAT. Data depicted that number of green leaves hill ${ }^{-}$ 1 increased gradually up to 60 DAT then started to decline.

Seeds treated with hormones were found to increase number of green leaves hill $^{-1}$ and maximum number of green leaves hill ${ }^{-1}$ were noted in kinetin primed set $\left(\mathrm{T}_{2}\right)$ which was followed by $\mathrm{T}_{1}$ and $\mathrm{T}_{3}$ 'over non primed control set under both sowing conditions but number of green leaves were found to decline under late sown condition. While non primed set $\left(\mathrm{T}_{0}\right)$ always showed poor performance. The trend was found similar for both the experimental years. Data regarding number of green leaves hill ${ }^{-1}$ was found statistically significant.

Table.1 Effect of hydro and hormonal seed priming on the plant height of rice (Var. HUR 105) under timely and late sown conditions

\begin{tabular}{|c|c|c|c|c|c|c|c|}
\hline \multirow[t]{2}{*}{ Year } & \multirow{2}{*}{$\begin{array}{l}\text { Sowing } \\
\text { Time }\end{array}$} & \multirow[t]{2}{*}{ Treatments*** } & \multicolumn{5}{|c|}{ Plant height $(\mathrm{cm})$} \\
\hline & & & 20 DAT & 40 DAT & 60 DAT & 80 DAT & 100 DAT \\
\hline \multirow{8}{*}{2015} & \multirow{4}{*}{ I* } & $\mathrm{T}_{0}$ & $41.10 \pm 0.52^{\mathrm{h}}$ & $62.00 \pm 0.5^{\mathrm{i}}$ & $71.50 \pm 0.29^{k}$ & $87.75 \pm 0.26^{\mathrm{i}}$ & $95.00 \pm 0.00^{\mathrm{h}}$ \\
\hline & & $\mathrm{T}_{1}$ & $46.80 \pm 0.12^{\mathrm{d}}$ & $70.25 \pm 0.43^{\mathrm{c}, \mathrm{d}}$ & $85.85 \pm 0.09^{c}$ & $106.60 \pm 0.92^{\mathrm{b}}$ & $107.00 \pm 0.00^{\mathrm{b}}$ \\
\hline & & $\mathrm{T}_{2}$ & $49.45 \pm 0.32^{b, c}$ & $73.40 \pm 0.35^{b}$ & $90.35 \pm 0.49^{\mathrm{a}, \mathrm{b}}$ & $105.85 \pm 0.26^{b}$ & $106.50 \pm 0.29^{b}$ \\
\hline & & $\mathrm{T}_{3}$ & $46.77 \pm 0.14^{\mathrm{d}}$ & $72.30 \pm 0.40^{b}$ & $89.10 \pm 2.37^{b}$ & $104.00 \pm 0.58^{\mathrm{c}}$ & $105.50 \pm 0.29^{c}$ \\
\hline & \multirow{4}{*}{$\mathbf{I I}^{* *}$} & $\mathrm{~T}_{0}$ & $38.60 \pm 0.23^{j}$ & $57.50 \pm 0.29^{j}$ & $69.25 \pm 0.43^{1}$ & $83.60 \pm 0.23^{\mathrm{k}}$ & $84.65 \pm 0.38^{\circ}$ \\
\hline & & $\mathrm{T}_{1}$ & $43.75 \pm 0.14^{\mathrm{g}}$ & $66.15 \pm 0.38^{\mathrm{g}}$ & $80.25 \pm 0.43^{\mathrm{i}}$ & $91.25 \pm 0.43^{\mathrm{g}}$ & $93.75 \pm 0.14^{\mathrm{i}}$ \\
\hline & & $\mathrm{T}_{2}$ & $47.20 \pm 0.46^{\mathrm{d}}$ & $67.70 \pm 0.40^{\mathrm{e}, \mathrm{f}}$ & $85.25 \pm 0.43^{\mathrm{c}, \mathrm{d}}$ & $91.70 \pm 0.17^{f}$ & $92.80 \pm 0.12^{j}$ \\
\hline & & $\mathrm{T}_{3}$ & $45.60 \pm 0.35^{\mathrm{e}}$ & $65.75 \pm 0.43^{\mathrm{g}}$ & $83.75 \pm 0.14^{\mathrm{d}, \mathrm{e}, \mathrm{f}}$ & $90.75 \pm 0.14^{\mathrm{g}}$ & $91.75 \pm 0.14^{\mathrm{k}}$ \\
\hline \multirow{8}{*}{2016} & \multirow{4}{*}{$\mathbf{I}^{*}$} & $\mathrm{~T}_{0}$ & $37.75 \pm 0.43^{\mathrm{k}}$ & $58.50 \pm 0.29^{j}$ & $69.20 \pm 0.46^{1}$ & $85.25 \pm 0.43^{\mathrm{g}}$ & $86.80 \pm 0.12^{\mathrm{n}}$ \\
\hline & & $\mathrm{T}_{1}$ & $43.80 \pm 0.12^{\mathrm{g}}$ & $67.75 \pm 0.14^{\mathrm{e}, \mathrm{f}}$ & $80.40 \pm 0.35^{\mathrm{i}}$ & $99.10 \pm 0.52^{\mathrm{j}}$ & $100.50 \pm 0.29^{\mathrm{g}}$ \\
\hline & & $\mathrm{T}_{2}$ & $46.95 \pm 0.55^{\mathrm{d}}$ & $69.90 \pm 0.52^{\mathrm{d}}$ & $81.75 \pm 0.14^{\mathrm{g}, \mathrm{h}, \mathrm{i}}$ & $100.70 \pm 0.75^{\mathrm{c}}$ & $102.50 \pm 0.29^{\mathrm{e}}$ \\
\hline & & $\mathrm{T}_{3}$ & $44.65 \pm 0.20^{f}$ & $66.80 \pm 0.12^{\mathrm{f}, \mathrm{g}}$ & $81.20 \pm 0.46^{\mathrm{h}, \mathrm{i}}$ & $100.40 \pm 0.92^{\mathrm{d}, \mathrm{e}}$ & $101.50 \pm 0.29^{f}$ \\
\hline & \multirow{4}{*}{ II** } & $\mathrm{T}_{0}$ & $32.75 \pm 0.14^{1}$ & $54.50 \pm 0.29^{k}$ & $66.10 \pm 0.06^{\mathrm{m}}$ & $81.75 \pm 0.14^{1}$ & $82.60 \pm 0.23^{\mathrm{P}}$ \\
\hline & & $\mathrm{T}_{1}$ & $39.70 \pm 0.17^{\mathrm{i}}$ & $63.65 \pm 0.20^{\mathrm{h}}$ & $75.90 \pm 0.12^{j}$ & $89.25 \pm 0.55^{\mathrm{h}}$ & $90.70 \pm 0.17^{1}$ \\
\hline & & $\mathrm{T}_{2}$ & $40.80 \pm 0.12^{\mathrm{h}}$ & $66.25 \pm 0.43^{\mathrm{g}}$ & $82.65 \pm 0.20^{\mathrm{f}, \mathrm{g}, \mathrm{h}}$ & $89.30 \pm 0.40^{\mathrm{h}}$ & $90.75 \pm 0.14^{1}$ \\
\hline & & $\mathrm{T}_{3}$ & $40.60 \pm 0.23^{\mathrm{h}}$ & $64.40 \pm 0.35^{\mathrm{h}}$ & $81.20 \pm 0.46^{\mathrm{g}, \mathrm{h,i}}$ & $88.40 \pm 0.35^{\mathrm{h}, \mathrm{i}}$ & $89.80 \pm 0.12^{\mathrm{m}}$ \\
\hline
\end{tabular}

Data presented are means from three replicates with standard errors. Within each treatment, different letters indicate significant differences by Duncan's multiple range test at $\mathrm{P}<0.05$

*Timely sown

** Late sown

*** $\mathrm{T}_{0}$ : Non primed control; $\mathrm{T}_{1}$ : Hydro primed; $\mathrm{T}_{2}$ : Kinetin and $\mathrm{T}_{3}$ : Salicylic acid 
Table.2 Effect of hydro and hormonal seed priming on number of tillers hill ${ }^{-1}$ of rice (Var. HUR 105) under timely and late sown conditions

\begin{tabular}{|c|c|c|c|c|c|c|c|}
\hline \multirow[t]{2}{*}{ Year } & \multirow{2}{*}{$\begin{array}{l}\text { Sowing } \\
\text { Time }\end{array}$} & \multirow[t]{2}{*}{ Treatments*** } & \multicolumn{4}{|c|}{ Number of tillers hill ${ }^{-1}$} & \multirow[b]{2}{*}{100 DAT } \\
\hline & & & 20 DAT & 40 DAT & 60 DAT & 80 DAT & \\
\hline \multirow{8}{*}{2015} & \multirow{4}{*}{$\mathbf{I}^{*}$} & $\mathrm{~T}_{0}$ & $7.33 \pm 0.33^{\mathrm{a}, \mathrm{b}, \mathrm{c}, \mathrm{d}}$ & $9.33 \pm 0.33^{b, c, d}$ & $10.67 \pm 0.33^{\mathrm{b}, \mathrm{c}}$ & $10.000 .58^{\mathrm{b}, \mathrm{c}}$ & $9.33 \pm 0.33^{\mathrm{c}}$ \\
\hline & & $\mathrm{T}_{1}$ & $8.67 \pm 0.88^{\mathrm{a}, \mathrm{b}}$ & $11.00 \pm 0.58^{\mathrm{a}, \mathrm{b}}$ & $12.67 \pm 0.33^{\mathrm{a}}$ & $11.67 \pm 0.33^{\mathrm{a}, \mathrm{b}}$ & $11.33 \pm 0.33^{\mathrm{a}, \mathrm{b}}$ \\
\hline & & $\mathrm{T}_{2}$ & $8.33 \pm 0.88^{\mathrm{a}, \mathrm{b}, \mathrm{c}}$ & $11.67 \pm 0.67^{\mathrm{a}}$ & $11.67 \pm 0.67^{\mathrm{a}, \mathrm{b}, \mathrm{c}}$ & $10.67 \pm 0.33^{a, b, c}$ & $10.00 \pm 0.58^{\mathrm{b}, \mathrm{c}}$ \\
\hline & & $\mathrm{T}_{3}$ & $8.00 \pm 1.00^{\mathrm{a}, \mathrm{b}, \mathrm{c}, \mathrm{d}}$ & $10.67 \pm 0.33^{a, b, c}$ & $11.33 \pm 0.33^{\mathrm{a}, \mathrm{b}, \mathrm{c}}$ & $10.33 \pm 0.67^{\mathrm{a}, \mathrm{b}, \mathrm{c}}$ & $10.33 \pm 0.33^{\mathrm{a}, \mathrm{b}, \mathrm{c}}$ \\
\hline & \multirow{4}{*}{ II** } & $\mathrm{T}_{0}$ & $6.33 \pm 0.67^{\mathrm{c}, \mathrm{d}}$ & $8.67 \pm 0.33^{\mathrm{c}, \mathrm{d}}$ & $10.33 \pm 0.33^{c}$ & $9.67 \pm 0.33^{c}$ & $9.33 \pm 0.33^{c}$ \\
\hline & & $\mathrm{T}_{1}$ & $7.33 \pm 0.33^{a, b, c, d}$ & $9.33 \pm 0.88^{b, c, d}$ & $11.67 \pm 0.33^{\mathrm{a}, \mathrm{b}, \mathrm{c}}$ & $11.00 \pm 0.00^{\mathrm{a}, \mathrm{b}, \mathrm{c}}$ & $10.67 \pm 0.33^{\mathrm{a}, \mathrm{b}, \mathrm{c}}$ \\
\hline & & $\mathrm{T}_{2}$ & $7.00 \pm 0.58^{\mathrm{a}, \mathrm{b}, \mathrm{c}, \mathrm{d}}$ & $9.67 \pm 0.88^{a, b, c, d}$ & $11.00 \pm 0.58^{\mathrm{a}, \mathrm{b}, \mathrm{c}}$ & $10.33 \pm 0.33^{\mathrm{a}, \mathrm{b}, \mathrm{c}}$ & $10.00 \pm 0.58^{\mathrm{b}, \mathrm{c}}$ \\
\hline & & $\mathrm{T}_{3}$ & $6.67 \pm 0.67^{\mathrm{b}, \mathrm{c}, \mathrm{d}}$ & $9.00 \pm 0.58^{\mathrm{b}, \mathrm{c}, \mathrm{d}}$ & $10.67 \pm 0.88^{b, c}$ & $10.33 \pm 0.33^{\mathrm{a}, \mathrm{b}, \mathrm{c}}$ & $10.00 \pm 0.58^{\mathrm{b}, \mathrm{c}}$ \\
\hline \multirow{8}{*}{2016} & \multirow{4}{*}{$I^{*}$} & $\mathrm{~T}_{0}$ & $6.00 \pm 0.58^{d}$ & $8.67 \pm 0.67^{c, d}$ & $11.00 \pm 0.58^{\mathrm{a}, \mathrm{b}, \mathrm{c}}$ & $10.67 \pm 0.67^{a, b, c}$ & $10.33 \pm 0.33^{\mathrm{a}, \mathrm{b}, \mathrm{c}}$ \\
\hline & & $\mathrm{T}_{1}$ & $7.00 \pm 0.58^{\mathrm{a}, \mathrm{b}, \mathrm{c}, \mathrm{d}}$ & $10.00 \pm 0.58^{\mathrm{a}, \mathrm{b}, \mathrm{c}, \mathrm{d}}$ & $11.67 \pm 0.33^{\mathrm{a}, \mathrm{b}, \mathrm{c}}$ & $11.33 \pm 0.33^{\mathrm{a}, \mathrm{b}, \mathrm{c}}$ & $11.67 \pm 0.33^{\mathrm{a}}$ \\
\hline & & $\mathrm{T}_{2}$ & $7.33 \pm 0.88^{\mathrm{a}, \mathrm{b}, \mathrm{c}, \mathrm{d}}$ & $9.33 \pm 0.88^{b, c, d}$ & $11.00 \pm 0.58^{\mathrm{a}, \mathrm{b}, \mathrm{c}}$ & $11.00 \pm 0.58^{\mathrm{a}, \mathrm{b}, \mathrm{c}}$ & $10.67 \pm 0.67^{\mathrm{a}, \mathrm{b}, \mathrm{c}}$ \\
\hline & & $\mathrm{T}_{3}$ & $6.67 \pm 0.88^{b, c, d}$ & $9.00 \pm 1.00^{b, c, d}$ & $10.67 \pm 0.33^{b, c}$ & $10.67 \pm 0.33^{a, b, c}$ & $10.33 \pm 0.33^{\mathrm{a}, \mathrm{b}, \mathrm{c}}$ \\
\hline & \multirow{4}{*}{ II** } & $\mathrm{T}_{0}$ & $6.33 \pm 0.33^{\mathrm{c}, \mathrm{d}}$ & $8.33 \pm 0.33^{\mathrm{d}}$ & $10.67 \pm 0.33^{b, c}$ & $10.67 \pm 0.33^{a, b, c}$ & $10.00 \pm 0.58^{\mathrm{b}, \mathrm{c}}$ \\
\hline & & $\mathrm{T}_{1}$ & $6.67 \pm 0.33^{b, c, d}$ & $9.67 \pm 0.33^{\mathrm{a}, \mathrm{b}, \mathrm{c}, \mathrm{d}}$ & $12.00 \pm 0.58^{\mathrm{a}, \mathrm{b}, \mathrm{c}}$ & $12.00 \pm 0.58^{\mathrm{a}}$ & $11.67 \pm 0.33^{\mathrm{a}}$ \\
\hline & & $\mathrm{T}_{2}$ & $6.67 \pm 0.33^{b, c, d}$ & $10.00 \pm 0.58^{\mathrm{a}, \mathrm{b}, \mathrm{c}, \mathrm{d}}$ & $11.67 \pm 0.33^{\mathrm{a}, \mathrm{b}, \mathrm{c}}$ & $11.67 \pm 0.33^{a, b}$ & $11.33 \pm 0.33^{\mathrm{a}, \mathrm{b}}$ \\
\hline & & $\mathrm{T}_{3}$ & $6.33 \pm 0.33^{\mathrm{c}, \mathrm{d}}$ & $9.33 \pm 0.33^{b, c, d}$ & $10.67 \pm 0.88^{b, c}$ & $10.67 \pm 0.88^{a, b, c}$ & $10.33 \pm 0.67^{\mathrm{a}, \mathrm{b}, \mathrm{c}}$ \\
\hline
\end{tabular}

Note: Detail of the conditions has given in table 1 .

Table.3 Effect of hydro and hormonal seed priming on number of green leaves hill-1 of rice (Var. HUR 105) under timely and late sown conditions

\begin{tabular}{|c|c|c|c|c|c|c|}
\hline \multirow[t]{2}{*}{ Year } & \multirow{2}{*}{$\begin{array}{l}\text { Sowing } \\
\text { Time }\end{array}$} & \multirow[t]{2}{*}{ Treatments*** } & \multicolumn{4}{|c|}{ Number of green leaves hill'-1 } \\
\hline & & & 20 DAT & 40 DAT & 60 DAT & 80 DAT \\
\hline \multirow{8}{*}{2015} & \multirow{4}{*}{$\mathbf{I} *$} & $\mathrm{~T}_{0}$ & $7.67 \pm 0.33^{\mathrm{d}, \mathrm{e}, \mathrm{f}, \mathrm{g}, \mathrm{h}}$ & $33.67 \pm 0.88^{\mathrm{c}, \mathrm{d}, \mathrm{e}}$ & $48.33 \pm 0.88^{\mathrm{g}, \mathrm{h}}$ & $46.33 \pm 0.88^{\mathrm{f}, \mathrm{g}}$ \\
\hline & & $\mathrm{T}_{1}$ & $10.33 \pm 0.88^{\mathrm{a}, \mathrm{b}}$ & $38.00 \pm 1.16^{\mathrm{a}, \mathrm{b}}$ & $55.00 \pm 1.16^{\mathrm{d}}$ & $50.00 \pm 0.58^{\mathrm{e}}$ \\
\hline & & $\mathrm{T}_{2}$ & $10.33 \pm 0.88^{\mathrm{a}, \mathrm{b}}$ & $39.33 \pm 1.77^{\mathrm{a}, \mathrm{b}}$ & $62.33 \pm 1.45^{\mathrm{a}, \mathrm{b}}$ & $57.67 \pm 1.45^{b, c}$ \\
\hline & & $\mathrm{T}_{3}$ & $8.00 \pm 0.58^{\mathrm{c}, \mathrm{d}, \mathrm{e}, \mathrm{f}, \mathrm{g}}$ & $36.67 \pm 1.45^{\mathrm{a}, \mathrm{b}, \mathrm{c}}$ & $60.00 \pm 1.16^{b, c}$ & $56.33 \pm 0.88^{\mathrm{c}, \mathrm{d}}$ \\
\hline & \multirow{4}{*}{ II** } & $\mathrm{T}_{0}$ & $6.33 \pm 0.88^{\mathrm{g}, \mathrm{h}}$ & $29.67 \pm 1.20^{f, g}$ & $43.33 \pm 0.88^{i, j}$ & $40.33 \pm 0.88^{h}$ \\
\hline & & $\mathrm{T}_{1}$ & $8.33 \pm 0.88^{\mathrm{c}, \mathrm{d}, \mathrm{e}, \mathrm{f}}$ & $32.33 \pm 1.45^{\mathrm{d}, \mathrm{e}, \mathrm{f}}$ & $49.00 \pm 0.58^{f, g, h}$ & $46.33 \pm 0.88^{\mathrm{f}, \mathrm{g}}$ \\
\hline & & $\mathrm{T}_{2}$ & $8.67 \pm 0.33^{\mathrm{b}, \mathrm{c}, \mathrm{d}, \mathrm{e}}$ & $33.33 \pm 0.88^{\mathrm{c}, \mathrm{d}, \mathrm{e}, \mathrm{f}}$ & $49.33 \pm 1.77^{\mathrm{f}, \mathrm{g}, \mathrm{h}}$ & $47.00 \pm 1.16^{\mathrm{f}, \mathrm{g}}$ \\
\hline & & $\mathrm{T}_{3}$ & $7.00 \pm 0.58^{\mathrm{e}, \mathrm{t}, \mathrm{g}, \mathrm{h}}$ & $30.33 \pm 0.88^{\mathrm{e}, \mathrm{t}, \mathrm{g}}$ & $47.67 \pm 1.45^{\mathrm{g}, \mathrm{h}}$ & $45.67 \pm 1.20^{\mathrm{g}}$ \\
\hline \multirow{8}{*}{2016} & \multirow{4}{*}{$\mathbf{I}^{*}$} & $\mathrm{~T}_{0}$ & $7.33 \pm 0.67^{\mathrm{e}, \mathrm{f}, \mathrm{g}, \mathrm{h}}$ & $32.33 \pm 1.45^{\mathrm{d}, \mathrm{e}, \mathrm{f}}$ & $46.33 \pm 0.88^{\mathrm{h}, \mathrm{i}}$ & $44.33 \pm 0.33^{\mathrm{g}}$ \\
\hline & & $\mathrm{T}_{1}$ & $9.33 \pm 0.33^{\mathrm{a}, \mathrm{b}, \mathrm{c}, \mathrm{d}}$ & $36.33 \pm 0.88^{a, b, c}$ & $52.67 \pm 1.45^{\mathrm{e}, \mathrm{f}}$ & $48.67 \pm 0.67^{\mathrm{e}, \mathrm{f}}$ \\
\hline & & $\mathrm{T}_{2}$ & $9.67 \pm 0.67^{a, b, c}$ & $38.33 \pm 1.20^{\mathrm{a}, \mathrm{b}}$ & $60.00 \pm 1.73^{b, c}$ & $58.00 \pm 1.16^{\mathrm{b}, \mathrm{c}}$ \\
\hline & & $\mathrm{T}_{3}$ & $8.33 \pm 0.33^{\mathrm{c}, \mathrm{d}, \mathrm{e}, \mathrm{f}}$ & $36.00 \pm 1.53^{b, c, d}$ & $57.67 \pm 1.45^{\mathrm{c}, \mathrm{d}}$ & $54.67 \pm 0.67^{d}$ \\
\hline & \multirow{4}{*}{ II** } & $\mathrm{T}_{0}$ & $6.00 \pm 0.58^{h}$ & $27.67 \pm 1.20^{\mathrm{g}}$ & $41.67 \pm 0.88^{j}$ & $38.67 \pm 0.67^{\mathrm{h}}$ \\
\hline & & $\mathrm{T}_{1}$ & $7.67 \pm 0.33^{\mathrm{d}, \mathrm{e}, \mathrm{f}, \mathrm{g}, \mathrm{h}}$ & $30.67 \pm 1.77^{\mathrm{e}, \mathrm{f}, \mathrm{g}}$ & $46.33 \pm 0.88^{h, i}$ & $44.67 \pm 0.67^{g}$ \\
\hline & & $\mathrm{T}_{2}$ & $8.00 \pm 0.58^{\mathrm{c}, \mathrm{d}, \mathrm{e}, \mathrm{f}, \mathrm{g}}$ & $31.33 \pm 0.88^{\mathrm{e}, \mathrm{f}, \mathrm{g}}$ & $47.33 \pm 1.77^{\mathrm{g}, \mathrm{h}}$ & $45.33 \pm 0.88^{\mathrm{g}}$ \\
\hline & & $\mathrm{T}_{3}$ & $6.67 \pm 0.33^{\mathrm{f,g}, \mathrm{h}}$ & $28.33 \pm 0.88^{\mathrm{g}}$ & $46.00 \pm 1.16^{\mathrm{h}, \mathrm{i}}$ & $44.33 \pm 0.88^{g}$ \\
\hline
\end{tabular}

Note: Detail of the conditions has given in table 
Table.4 Effect of hydro and hormonal seed priming on leaf area hill ${ }^{-1}$ of rice (Var. HUR 105) under timely and late sown conditions

\begin{tabular}{|c|c|c|c|c|c|c|}
\hline \multirow[t]{2}{*}{ Year } & \multirow{2}{*}{$\begin{array}{l}\text { Sowing } \\
\text { Time }\end{array}$} & \multirow[t]{2}{*}{ Treatments*** } & \multicolumn{3}{|c|}{ Leaf area hill ${ }^{-1}\left(\mathrm{~cm}^{2}\right)$} & \multirow[b]{2}{*}{80 DAT } \\
\hline & & & 20 DAT & 40 DAT & 60 DAT & \\
\hline \multirow{8}{*}{2015} & \multirow{4}{*}{ I* } & $\mathrm{T}_{0}$ & $238.33 \pm 4.41^{\mathrm{g}, \mathrm{h}, \mathrm{i}}$ & $697.67 \pm 9.83^{\mathrm{g}, \mathrm{h}, \mathrm{i}}$ & $1075.43 \pm 5.41^{\mathrm{h}, \mathrm{I}, \mathrm{j}}$ & $850.53 \pm 9.08^{\mathrm{e}}$ \\
\hline & & $\mathrm{T}_{1}$ & $269.33 \pm 6.37^{\mathrm{b}, \mathrm{c}, \mathrm{d}, \mathrm{e}, \mathrm{f}}$ & $733.67 \pm 5.55^{\mathrm{a}, \mathrm{b}, \mathrm{c}, \mathrm{d}, \mathrm{e}}$ & $1166.00 \pm 4.05^{\mathrm{a}, \mathrm{b}, \mathrm{c}, \mathrm{d}}$ & $893.33 \pm 3.72^{c}$ \\
\hline & & $\mathrm{T}_{2}$ & $273.57 \pm 3.57^{\mathrm{b}, \mathrm{c}, \mathrm{d}, \mathrm{e}}$ & $720.67 \pm 2.85^{\mathrm{c}, \mathrm{d}, \mathrm{e}, \mathrm{f}}$ & $1157.50 \pm 3.41^{\mathrm{b}, \mathrm{c}, \mathrm{d}, \mathrm{e}}$ & $963.67 \pm 6.23^{\mathrm{a}, \mathrm{b}}$ \\
\hline & & $\mathrm{T}_{3}$ & $256.33 \pm 11.27^{\mathrm{d}, \mathrm{e}, \mathrm{f}, \mathrm{g}}$ & $737.00 \pm 7.10^{\mathrm{a}, \mathrm{b}, \mathrm{c}, \mathrm{d}}$ & $1093.67 \pm 2.97^{\mathrm{g}}$ & $882.67 \pm 6.57^{\mathrm{c}, \mathrm{d}}$ \\
\hline & \multirow{4}{*}{ II** } & $\mathrm{T}_{0}$ & $232.33 \pm 5.18^{\mathrm{h}, \mathrm{i}}$ & $691.00 \pm 8.51^{\mathrm{h}, \mathrm{i}}$ & $1063.83 \pm 6.57^{\mathrm{j}, \mathrm{k}}$ & $846.97 \pm 6.22^{\mathrm{e}}$ \\
\hline & & $\mathrm{T}_{1}$ & $262.57 \pm 6.86^{\mathrm{c}, \mathrm{d}, \mathrm{e}, \mathrm{f}}$ & $726.40 \pm 5.56^{\mathrm{b}, \mathrm{c}, \mathrm{d}, \mathrm{e}}$ & $1155.07 \pm 5.86^{\mathrm{b}, \mathrm{c}, \mathrm{d}, \mathrm{e}}$ & $884.50 \pm 3.28^{\mathrm{c}, \mathrm{d}}$ \\
\hline & & $\mathrm{T}_{2}$ & $263.57 \pm 5.23^{\mathrm{c}, \mathrm{d}, \mathrm{e}, \mathrm{f}}$ & $712.10 \pm 5.08^{\mathrm{e}, \mathrm{f}, \mathrm{g}, \mathrm{h}}$ & $1147.43 \pm 1.82^{\mathrm{e}, \mathrm{f}}$ & $952.43 \pm 6.61^{\mathrm{a}, \mathrm{b}}$ \\
\hline & & $\mathrm{T}_{3}$ & $246.93 \pm 11.07^{\mathrm{f}, \mathrm{g}, \mathrm{h}}$ & $726.90 \pm 8.02^{\mathrm{a}, \mathrm{b}, \mathrm{c}, \mathrm{d}, \mathrm{e}}$ & $1081.83 \pm 2.92^{\mathrm{g}, \mathrm{h}, \mathrm{i}}$ & $874.97 \pm 7.32^{\mathrm{c}, \mathrm{d}}$ \\
\hline \multirow{8}{*}{2016} & \multirow{4}{*}{$\mathbf{I}^{*}$} & $\mathrm{~T}_{0}$ & $232.33 \pm 3.72^{h, i}$ & $691.43 \pm 9.98^{\mathrm{h}, \mathrm{i}}$ & $1066.50 \pm 5.97^{\mathrm{i}, \mathrm{j}, \mathrm{k}}$ & $848.50 \pm 10.21^{\mathrm{e}}$ \\
\hline & & $\mathrm{T}_{1}$ & $267.27 \pm 4.30^{\mathrm{c}, \mathrm{d}, \mathrm{e}, \mathrm{f}}$ & $730.67 \pm 6.17^{\mathrm{a}, \mathrm{b}, \mathrm{c}, \mathrm{d}, \mathrm{e}}$ & $1161.27 \pm 2.26^{\mathrm{b}, \mathrm{c}, \mathrm{d}, \mathrm{e}}$ & $886.27 \pm 2.46^{\mathrm{c}, \mathrm{d}}$ \\
\hline & & $\mathrm{T}_{2}$ & $268.60 \pm 3.71^{\mathrm{c}, \mathrm{d}, \mathrm{e}, \mathrm{f}}$ & $714.57 \pm 3.30^{\mathrm{d}, \mathrm{e}, \mathrm{f}, \mathrm{g}}$ & $1151.10 \pm 2.48^{\mathrm{d}, \mathrm{e}, \mathrm{f}}$ & $958.73 \pm 7.48^{a, b}$ \\
\hline & & $\mathrm{T}_{3}$ & $250.73 \pm 10.70^{\mathrm{e}, \mathrm{f}, \mathrm{g}, \mathrm{h}}$ & $735.57 \pm 8.51^{\mathrm{a}, \mathrm{b}, \mathrm{c}, \mathrm{d}}$ & $1084.33 \pm 2.69^{\mathrm{g}, \mathrm{h}}$ & $876.67 \pm 5.04^{\mathrm{c}, \mathrm{d}}$ \\
\hline & \multirow{4}{*}{ II** } & $\mathrm{T}_{0}$ & $223.67 \pm 5.24^{1}$ & $681.83 \pm 6.84^{1}$ & $1057.57 \pm 5.92^{\mathrm{k}}$ & $840.03 \pm 5.78^{\mathrm{e}}$ \\
\hline & & $\mathrm{T}_{1}$ & $256.10 \pm 10.17^{\mathrm{d}, \mathrm{e}, \mathrm{f}, \mathrm{g}}$ & $700.87 \pm 4.34^{\mathrm{f}, \mathrm{g}, \mathrm{h}, \mathrm{i}}$ & $1139.50 \pm 2.85^{\mathrm{f}}$ & $876.53 \pm 6.28^{c, d}$ \\
\hline & & $\mathrm{T}_{2}$ & $257.23 \pm 5.86^{\mathrm{d}, \mathrm{e}, \mathrm{f}, \mathrm{g}}$ & $725.67 \pm 1.57^{\mathrm{b}, \mathrm{c}, \mathrm{d}, \mathrm{e}}$ & $1148.40 \pm 5.98^{\mathrm{e}, \mathrm{f}}$ & $947.47 \pm 9.03^{b}$ \\
\hline & & $\mathrm{T}_{3}$ & $246.83 \pm 5.90^{\mathrm{fg}, \mathrm{h}}$ & $720.30 \pm 2.67^{c, d, e}$ & $1074.17 \pm 3.17^{\mathrm{h}, \mathrm{I}, \mathrm{j}, \mathrm{k}}$ & $871.00 \pm 6.11^{\mathrm{d}}$ \\
\hline
\end{tabular}

Note: Detail of the conditions has given in table

Table.5 Effect of hydro and hormonal seed priming on leaf area index (LAI) of rice (Var. HUR 105) under timely and late sown conditions

\begin{tabular}{|c|c|c|c|c|c|c|}
\hline \multirow[t]{2}{*}{ Year } & \multirow{2}{*}{$\begin{array}{l}\text { Sowing } \\
\text { Time }\end{array}$} & \multirow[t]{2}{*}{ Treatments*** } & \multicolumn{4}{|c|}{ LAI } \\
\hline & & & 20 DAT & 40 DAT & 60 DAT & 80 DAT \\
\hline \multirow{8}{*}{2015} & \multirow{4}{*}{$\mathbf{I}^{*}$} & $\mathrm{~T}_{0}$ & $0.60 \pm 0.01^{\mathrm{c}, \mathrm{d}}$ & $1.74 \pm 0.10^{\mathrm{e}}$ & $2.69 \pm 0.06^{\mathrm{c}, \mathrm{d}}$ & $2.13 \pm 0.12^{\mathrm{c}, \mathrm{d}, \mathrm{e}}$ \\
\hline & & $\mathrm{T}_{1}$ & $0.64 \pm 0.04^{b, c}$ & $1.80 \pm 0.07^{\mathrm{d}}$ & $2.89 \pm 0.11^{\mathrm{a}, \mathrm{b}}$ & $2.21 \pm 0.10^{\mathrm{c}, \mathrm{d}}$ \\
\hline & & $\mathrm{T}_{2}$ & $0.69 \pm 0.01^{\mathrm{a}, \mathrm{b}}$ & $1.85 \pm 0.07^{\mathrm{a}, \mathrm{b}}$ & $2.92 \pm 0.06^{\mathrm{a}}$ & $2.41 \pm 0.10^{\mathrm{a}}$ \\
\hline & & $\mathrm{T} 3$ & $0.68 \pm 0.06^{\mathrm{a}, \mathrm{b}}$ & $1.84 \pm 0.10^{\mathrm{a}, \mathrm{b}, \mathrm{c}}$ & $2.73 \pm 0.11^{\mathrm{c}}$ & $2.23 \pm 0.12^{\mathrm{c}, \mathrm{d}}$ \\
\hline & \multirow{4}{*}{ II** } & $\mathrm{T}_{0}$ & $0.60 \pm 0.01^{\mathrm{c}, \mathrm{d}}$ & $1.73 \pm 0.07^{\mathrm{e}}$ & $2.66 \pm 0.09^{\mathrm{d}, \mathrm{e}}$ & $2.12 \pm 0.08^{\mathrm{c}, \mathrm{d}, \mathrm{e}}$ \\
\hline & & $\mathrm{T}_{1}$ & $0.63 \pm 0.01^{b, c}$ & $1.78 \pm 0.10^{\mathrm{d}}$ & $2.87 \pm 0.06^{\mathrm{a}, \mathrm{b}}$ & $2.19 \pm 0.08^{c, d}$ \\
\hline & & $\mathrm{T}_{2}$ & $0.67 \pm 0.03^{b}$ & $1.83 \pm 0.07^{b, c}$ & $2.89 \pm 0.06^{\mathrm{a}, \mathrm{b}}$ & $2.38 \pm 0.12^{\mathrm{a}, \mathrm{b}}$ \\
\hline & & $\mathrm{T}_{3}$ & $0.66 \pm 0.05^{b}$ & $1.82 \pm 0.10^{b, c}$ & $2.70 \pm 0.06^{\mathrm{c}, \mathrm{d}}$ & $2.21 \pm 0.12^{\mathrm{c}, \mathrm{d}}$ \\
\hline \multirow{8}{*}{2016} & \multirow{4}{*}{$\mathbf{I}^{*}$} & $\mathrm{~T}_{0}$ & $0.58 \pm 0.08^{d}$ & $1.73 \pm 0.10^{\mathrm{e}}$ & $2.67 \pm 0.08^{\mathrm{c}, \mathrm{d}}$ & $2.12 \pm 0.12^{\mathrm{c}, \mathrm{d}, \mathrm{e}}$ \\
\hline & & $\mathrm{T}_{1}$ & $0.63 \pm 0.12^{b, c}$ & $1.79 \pm 0.05^{\mathrm{c}, \mathrm{d}}$ & $2.88 \pm 0.10^{\mathrm{a}, \mathrm{b}}$ & $2.19 \pm 0.06^{\mathrm{c}, \mathrm{d}}$ \\
\hline & & $\mathrm{T}_{2}$ & $0.68 \pm 0.05^{\mathrm{a}, \mathrm{b}}$ & $1.84 \pm 0.10^{\mathrm{a}, \mathrm{b}}$ & $2.90 \pm 0.12^{\mathrm{a}, \mathrm{b}}$ & $2.40 \pm 0.06^{\mathrm{a}}$ \\
\hline & & $\mathrm{T}_{3}$ & $0.67 \pm 0.05^{\mathrm{a}, \mathrm{b}}$ & $1.83 \pm 0.09^{\mathrm{a}, \mathrm{b}, \mathrm{c}}$ & $2.71 \pm 0.10^{\mathrm{c}, \mathrm{d}}$ & $2.22 \pm 0.12^{\mathrm{c}, \mathrm{d}}$ \\
\hline & \multirow{4}{*}{$\mathbf{I I}^{* *}$} & $\mathrm{~T}_{0}$ & $0.56 \pm 0.08^{d}$ & $1.70 \pm 0.05^{\mathrm{e}, \mathrm{f}}$ & $2.64 \pm 0.08^{\mathrm{c}, \mathrm{d}}$ & $2.10 \pm 0.10^{\mathrm{c}, \mathrm{d}, \mathrm{e}}$ \\
\hline & & $\mathrm{T}_{1}$ & $0.62 \pm 0.05^{b, c}$ & $1.75 \pm 0.09^{\mathrm{d}, \mathrm{e}}$ & $2.69 \pm 0.12^{\mathrm{c}, \mathrm{d}}$ & $2.18 \pm 0.10^{\mathrm{c}, \mathrm{d}}$ \\
\hline & & $\mathrm{T}_{2}$ & $0.66 \pm 0.08^{b, c}$ & $1.81 \pm 0.09^{d}$ & $2.87 \pm 0.10^{\mathrm{a}, \mathrm{b}}$ & $2.37 \pm 0.12^{b}$ \\
\hline & & $\mathrm{T}_{3}$ & $0.64 \pm 0.05^{b, c}$ & $1.80 \pm 0.05^{\mathrm{d}}$ & $2.85 \pm 0.12^{\mathrm{a}, \mathrm{b}}$ & $2.19 \pm 0.06^{\mathrm{c}, \mathrm{d}}$ \\
\hline
\end{tabular}




\section{Leaf area hill ${ }^{-1}$}

Data regarding leaf area hill $^{-1}$ is represented in table 4, measured at 20, 40, 60 and 80 DAT. It was noted that leaf area was found to increase till 60 DAT, after that reduction was noted. It was observed from the data that during late sown condition leaf area was found decreased. However primed sets were found to increase leaf area under both the sowing conditions and maximum leaf area hill $^{-1}$ was noted in kinetin primed set $\left(\mathrm{T}_{2}\right)$ followed by $T_{1}$ and data was found statistically significant for each study period and both the studied years

\section{Leaf area Index}

Leaf area index (LAI) of rice Var. HUR 105 is represented in table 5, measured at 20, 40, 60 and 80 DAT. Leaf area index was found to increase till 60 DAT, after that reduction in LAI was noted. It was also noted that during late sown condition leaf area index was found to decrease but primed seeds exhibited good performance under both the sowing conditions (Timely and late) and maximum LAI was noted in primed set $T_{2}$ followed by $T_{3}$ and $T_{1}$. The same pattern was followed in both studied years and statistical analysis presented significant differences in primed sets in respect to non primed set.

Plant height of rice, as affected by primed and non primed seeds of variety HUR 105 is presented in table 1 . It has been cleared that the hydro and kinetin primed seeds always improved the plant height as compared to non primed one but hydro and Salicylic acid primed set showed less values and reduction in plant height was noted in late sown condition. Similar result was noted by Bose et al., 2007 and Safdar et al.2013. Study regarding the number of tillers hill ${ }^{-1}$ suggested that the hormone primed sets have more tiller number hill ${ }^{-1}$ with respect to non primed sets.
The kinetin treated sets sowed best result within primed sets. Seed priming of rice resulted in early and uniform emergence of healthy and vigorous seedlings which gave a vigorous start and seedling establishment. Kant et al., (2006) reported, seed priming with water, salts and growth regulator resulted in more tillers, higher dry matter in leaves, stem and reproductive parts, and better grain yield of wheat. They also found that if seed priming done with indole- 3- acetic acid, $\mathrm{KCl}$, water, $\mathrm{ZnSO}_{4}$ and $\mathrm{Na}_{2} \mathrm{SO}_{4}$ resulted more plant dry matter and grain yield than non primed set. Zheng et al., (2002) reported that seed priming significantly increase tiller number in rice and wheat. The beneficial effects of salts like $\mathrm{MgSO}_{4}, \mathrm{Mg}\left(\mathrm{NO}_{3}\right)_{2}, \mathrm{CaCl}_{2}$ etc. were seen on plant height, leaf number and leaf area of mustard, rice crops and wheat (Pandey and Bose 2006, Srivastava and Bose 2012; Hussian et al., 2013) and similar result was noted in present case.

The present study concludes that kinetin treatment in form of seed priming can improve the plant growth and development in normal as well as late sown conditions of rice var: HUR 105. Therefore it can be suggested that the late sowing affect may be ameliorated with this type of treatment.

\section{References}

Baloch, M.S., Awan, I.U. and Hassan, G., 2006. Growth and yield of rice as affected by transplanting dates and seedlings per hill under high temperature of Dera Ismail Khan, Pakistan. Journal of Zhejiang University Science B, 7(7), pp.572-579.

Bose B Kumar R Kuril S K and Srivastava A K (2007) Hardening of mustard seeds with magnesioum nitrate increases seed germination, vegetative growth, nitrogen assimilation and yield. Brassica 9 (14): 3338.

Chen, Keting, Arora R (2011) Dynamics of the antioxidant system during seed osmopriming, post-priming germination, and seedling 
establishment in Spinach (Spinacia oleracea). Plant Science. 180 :212-220.

Farooq, M., Basra, S.M.A., Rehman, H. and Saleem, B.A., 2008. Seed priming enhances the performance of late sown wheat (Triticum aestivum L.) by improving chilling tolerance. Journal of Agronomy and Crop Science, 194(1), pp.55-60.

Hussian, I., Ahmad, R., Farooq, M. and Wahid, A., 2013. Seed priming improves the performance of poor quality wheat seed. International Journal of Agriculture \& Biology, 15(6).

Iqbal, M. and Ashraf, M., 2007. Seed Treatment with Auxins Modulates Growth and Ion Partitioning in Salt- stressed Wheat Plants. Journal of Integrative Plant Biology, 49(7), pp.1003-1015.

Kant, S., Pahuja, S.S. and Pannu, R.K., 2004. Effect of seed priming on growth and phenology of wheat under late- sown conditions. Tropical science, 44(1), pp.9-15.

Korkmaz, Ahmet, Korkmaz Y (2009) Promotion by 5 -aminolevulenic acid of pepper seed germination and seedling emergence under low-temperature stress. Scientia horticulturae. 119: 98-102

Krishnotar, Bose B, Srivastava AK, Shahi JP (2009) Response of rabi maize crop to seed invigoration with magnes ium nitrate and distilled water. Indian J Plant Physiol. 14:7177.

Kumar, M., Pant, B., Mondal, S. and Bose, B. 2016. Hydro and halo priming: influenced germination responses in wheat Var-HUW468 under heavy metal stress. Acta physiologiae plantarum. 38:217.

Lichtfouse, E., 2009. Organic farming, pest control and remediation of soil pollutants (Vol. 1, p. 418). Springer.

Mahajan, G., Sarlach, R.S., Japinder, S. and Gill, M.S., 2011. Seed priming effects on germination, growth and yield of dry directseeded rice. Journal of crop improvement, 25(4), pp.409-417.

Pandey, D. and Bose, B., 2006. Influence of presowing seed treatment with nitrate salts and different sowing dates on performance of mustard. Indian Journal of Plant Physiology, 11(3), p.261.

Pant B, Bose B (2016) Mitigation of the influence of PEG-6000 imposed water stress on germination of halo primed rice seeds. International Journal of Agriculture, Environment \& Biotechnology, 9:275.

Safdar, M.E., Noorka, I.R., Tanveer, A., Tariq, S.A. and Rauf, S., 2013. Growth and yield of advanced breeding lines of médium grain rice as influenced by different transplanting dates. The Journal of Animal \& Plant Sciences, 23(1), pp.227-231.

Srivastava AK, Bose B (2012) Effect of nitrate seed priming on Phenology, crop growth rate and yield attributes in rice (Oryza sativa L.). Vegetos. 25:174-181

Watson, D.J., 1947. Comparative physiological studies on the growth of field crops: I. Variation in net assimilation rate and leaf area between species and varieties, and within and between years. Annals of botany, 11(41), pp.41-76.

Zheng, H.C., 2002. Effect of seed priming with mixed-salt solution on emergence and physiological characteristics of seedling in rice (Oryza sativa L.) under stress conditions. J Zhejiang Univ (Agric \& Life Sci), 28, pp.175-178.

Zhou ZS, Guo K, Elbaz AA, Yang ZM (2009) Salicylic acid alleviate mercury toxicity by preventing oxidative stress in roots of Medicago sativa. Environ Exp Bot 65:27-34

\section{How to cite this article:}

Mahesh Kumar, Rajesh Kumar Singhal, Bandana Bose. 2018. Effect of Hydro and Hormonal Priming on Growth and Development of Rice under Timely and Late Sown Conditions. Int.J.Curr.Microbiol.App.Sci. 7(05): 2970-2976.

doi: https://doi.org/10.20546/ijcmas.2018.705.345 